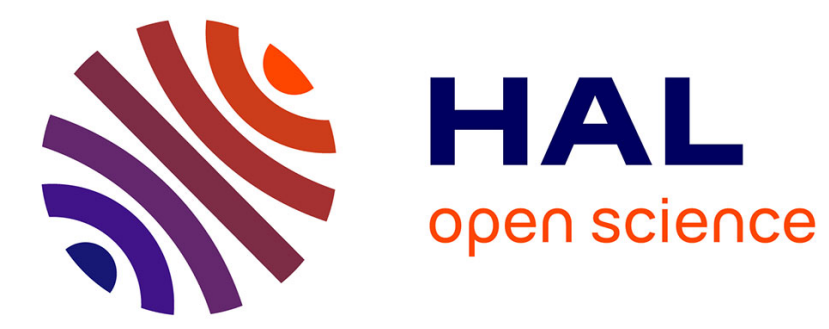

\title{
Critical behaviour for vulcanization processes
}

P.-G. de Gennes

\section{To cite this version:}

P.-G. de Gennes. Critical behaviour for vulcanization processes. Journal de Physique Lettres, 1977, 38 (17), pp.355-358. 10.1051/jphyslet:019770038017035500 . jpa-00231395

\section{HAL Id: jpa-00231395 https://hal.science/jpa-00231395}

Submitted on 1 Jan 1977

HAL is a multi-disciplinary open access archive for the deposit and dissemination of scientific research documents, whether they are published or not. The documents may come from teaching and research institutions in France or abroad, or from public or private research centers.
L'archive ouverte pluridisciplinaire HAL, est destinée au dépôt et à la diffusion de documents scientifiques de niveau recherche, publiés ou non, émanant des établissements d'enseignement et de recherche français ou étrangers, des laboratoires publics ou privés. 


\title{
CRITICAL BEHAVIOUR FOR VULCANIZATION PROCESSES
}

\author{
P. G. DE GENNES \\ Collège de France, 75231 Paris Cedex 05, France (*) \\ (Reçu le 23 juin 1977, accepté le 4 août 1977)
}

\begin{abstract}
Résumé. - Nous considérons un système de chaînes fondues, avec $N$ monomères/chaîne et une fraction $\rho$ des monomères pontés par un agent de vulcanisation. Au voisinage de la transition solgel $\left(\rho=\rho_{\mathrm{c}} / \Delta \rho\right)$ nous trouvons que la théorie de Flory est acceptable à $N>\left(\rho_{\mathrm{c}} / \Delta \rho\right)^{3}$. Pour des chaînes plus courtes on doit retrouver les exposants critiques de la percolation.

Abstract. - We consider a polymer melt, with polymerization index $N$ and a fraction $\rho$ of cross linked monomers, for values of $\rho$ close to the gelation threshold $\left(\rho=\rho_{\mathrm{c}}+\Delta \rho\right)$. Using a Ginsburg argument, we find that (in three dimensions) the Flory theory is qualitatively correct whenever $N>\left(\rho_{\mathrm{c}} / \Delta \rho\right)^{3}$. For shorter chains the critical exponents are expected to be of the percolation type.
\end{abstract}

1. Introduction. - The classical theory of gelation for branched polycondensates $[1,2]$ is based on a tree approximation : the trees are generated by a random branching process. Cyclizations are either neglected, or included only at the level of short cycles. This approach gives rather good numerical values for the gelation threshold $\rho_{\mathrm{c}}$. But it is qualitatively incorrect for all critical exponents describing the behaviour near threshold $[5,6]$. The critical exponents are in fact known from numerical studies on the related percolation problem [7] :

a) the gel fraction $\theta$, as a function of the fraction $\rho$ of reacted bonds is expected to behave (near threshold) like :

$$
\theta \cong\left(\frac{\rho-\rho_{\mathrm{c}}}{\rho_{\mathrm{c}}}\right)^{\beta}=\left(\frac{\Delta \rho}{\rho_{\mathrm{c}}}\right)^{\beta} \quad \beta=0.39
$$

b) the correlation length $\xi(\rho)$ behaves like

$$
\xi=\xi_{0}\left(\frac{\Delta \rho}{\rho_{\mathrm{c}}}\right)^{-v} \quad v \simeq 0.9
$$

c) the average polymerization index $Z$ of the finite clusters diverges like

$$
Z=\left(\frac{\Delta \rho}{\rho_{\mathrm{c}}}\right)^{-\gamma} \gamma=1.8
$$

These exponents differ strongly from the predictions of the tree approximation

$$
\beta_{\mathrm{t}}=1 \quad \gamma_{\mathrm{t}}=1 \quad v_{\mathrm{t}}=1 / 2
$$

(*) Also : Ecole de Physique et de Chimie, Paris.
In the present note, we discuss another sol-gel transition : the vulcanization process, where a system of flexible chains, of large polymerization index $N$, becomes cross linked through the action of small agents such as a sulphur atom [3, 4]. We have discussed in the past some features of vulcanization between dilute, ideal chains in solution [8]. In the present note, we come to a much more practical problem, where the chains form a concentrated solution and have realistic interactions. We shall show that, for large $N$, and for three dimensional systems, the critical exponents of the vulcanization transition have their classical values (eq. (1.4)) : the tree approximation is in better shaps for long chains than for the branching of small units.

We have reached this result by two methods :

a) direct discussion of fluctuations inside one correlation volume (section 2),

b) introduction of a generalized Potts model which defines a correspondence between vulcanization and a certain type of magnetic phase transitions (section 3 ).

Method $(a)$ is more accessible to physical chemists, while method $(b)$ is rather directed towards solid state physicists.

2. Direct Ginsburg argument. - The deviations from mean field exponents are due to fluctuations in the number of connected monomers inside one correlation volume. We shall now estimate three fluctuations following the spirit of an early argument by Ginsburg for phase transitions [9] :

a) we compute the fluctuations assuming that the classical exponents are valid,

b) we compare them to the average gel fraction $\theta$ : whenever the fluctuations are small with respect to $\theta$, 
we conclude that the tree approximation is qualitatively correct. All our arguments will be written for an arbitrary space dimensionality $d$.

We consider a volume $\Omega=\xi^{d}$ containing an average number of monomers

$$
v_{\text {tot }}=c_{0} \Omega .
$$

(where $c_{0} \sim a^{-d}$ is the monomer concentration). We choose a reaction level $\rho$ slightly larger than the threshold $\rho_{\mathrm{c}}$ : then we have an average number $v_{\mathrm{gel}}$ of monomers inside $\Omega$, belonging to the gel fraction

$$
v_{\mathrm{gel}}=c_{0} \theta \Omega \text {. }
$$

The fluctuations of $v_{\text {tot }}$ are negligible. Thus the fluctuations of $v_{\mathrm{gel}}$ and those of $v_{\mathrm{fc}}$ (number of monomers belonging to finite clusters) are related by

$$
\Delta v_{\mathrm{gel}}+\Delta v_{\mathrm{fc}}=0
$$

In a volume $\Omega$ we have $v_{\mathrm{fc}}$ monomers grouped into $g=v_{\mathrm{fc}} / Z$ clusters, where $Z$ is the average number of monomers/clusters. In the free approximation

$$
Z \cong N \rho_{\mathrm{c}} / \Delta \rho
$$

Thus we expect relative fluctuations

$$
\left(\frac{\Delta v_{\mathrm{fc}}}{v_{\mathrm{fc}}}\right)^{2} \sim \frac{1}{g}=\frac{Z}{v_{\mathrm{fc}}}
$$

or since $v_{\mathrm{fc}}$ is nearly equal to $v_{\mathrm{tot}}(\theta \rightarrow 0)$

$$
\left\langle\Delta v_{\mathrm{gel}}^{2}\right\rangle=\left\langle\Delta v_{\mathrm{fc}}^{2}\right\rangle \cong v_{\mathrm{tot}} Z .
$$

The relative importance of the fluctuations is measured by the quantity

$$
X=\left(\Delta v_{\mathrm{gel}} / v_{\mathrm{gel}}\right)^{2} \cong Z /\left(\theta^{2} c_{0} \xi^{d}\right)
$$

We now insert for $Z, \theta$, and $\xi$ the classical exponents of eq. (1.4). One coefficient is important : the prefactor $\xi_{0}$ in eq. (1.2) is of the order of the size on one chain. For a dense system we know both from theory [1] and from neutron experiments [10] that the chains are ideal : thus

$$
\xi_{0} \cong N^{1 / 2} a
$$

We also note that $\rho_{\mathrm{c}} \sim 1 / N$ as explained in reference [4]. Then

$$
X \cong N^{1-d / 2}\left(\rho_{\mathrm{c}} / \Delta \rho\right)^{3-d / 2} .
$$

a) Consider first the usual case $(d=3)$. Then eq. (2.8) shows that $X$ is small except in a very narrow range $|\Delta v| \lesssim \Delta \rho^{*}$

$$
\frac{\Delta \rho^{*}}{\rho_{\mathrm{c}}} \sim N^{-1 / 3} \quad(d=3) .
$$

Thus for $N=10^{3}$ the classical exponents should hold except for a small interval of order $1 / 10 \rho_{c}$ (where the system crosses over towards the percolation indices).

b) Eq. (2.8) shows that for $d \geqslant 6$ the fluctuations are always weak as is known for percolation [11].

c) For $d=2, X=\left[\rho_{\mathrm{c}} / \Delta \rho\right]^{2}$ is large in all the critical region and the percolation indices must be observed. This could possibly be studied on monomolecular films.

3. A Potts model for vulcanization. - The $s$-state Potts model is a generalization of the Ising model of magnetism where each magnetic moment can be in $s$ different states [12]. Kastelejn and Fortuin have shown that taking the formal limit $s \rightarrow 1$ in the Potts model, one generates a percolation transition [13]. In the present section, we construct a slight generalization of the Potts model which, in the same limit $s \rightarrow 1$, generates a vulcanization problem. We then show how the Ginsburg criterion can be discussed in this representation.

Let us start with a random set of chains, represented by a set of non-intersecting random walks on a periodic lattice. We consider only concentrated systems ; each lattice site carries one and only one monomer.

To each lattice site $(i)$ we associate a spin $\mathbf{S}_{i}$ : this is a classical vector in an $s-1$ dimensional space; it can take $s$ different positions at the corners of a regular polyhedron [14].

For a given arrangement of the chains, we introduce a hamiltonian $\mathcal{H}$ describing certain couplings $J_{i j}$ between nearest neighbour spins

$$
\mathscr{H}=-\sum_{i<j} J_{i j} \mathbf{S}_{i} \cdot \mathbf{S}_{j}
$$

$J_{i j}=\left\{\begin{array}{l}J_{0} \text { if }(i, j) \text { belong to the same chain } \\ J \text { if }(i, j) \text { belong to different chains }\end{array}\right\}$

$$
J_{0} \gg N J>0 \text {. }
$$

To the system described by (3.1) we associate a fictitious temperature $\tau$ (which has nothing to do with the physical temperature of the chains) and we restrict our attention to the temperature region

$$
\tau \sim N J \ll J_{0} .
$$

Then all spins along one chain are locked and parallel to each other.

From reference [13] we know that if we take the formal limit $s \rightarrow 1$ in the system described by (3.1), we generate a percolation problem, where two nearest neighbour sites $i$ and $j$ have a probability $p_{i j}$ of being connected

$$
p_{i j}=1-\exp \left(-2 J_{i j} / \tau\right)
$$


With our choice of $J_{i j}$, and the inequality (3.2), we see that

$p_{i j}=\left\{\begin{array}{l}1 \text { if }(i) \text { and }(j) \text { belong to the same chain } \\ p=1-\exp -\frac{2 J}{\tau} \cong \frac{2 J}{\tau} \text { if not } .\end{array}\right.$

This corresponds exactly to a vulcanization problem, where the cross-linking species is spread over all bonds between different chains with a (small) probability $p$ of building up a cross link : the fraction of cross linked monomers $\rho$ and the probability $p$ are proportional $\left[\rho \cong p\left(Z_{1}-2\right)\right.$, where $Z_{1}$ is the coordination number of the lattice].

For the temperature interval of interest, the Landau Ginsburg free energy [14] corresponding to eq. (3.1) has the form

$$
F / \tau=A\left[\frac{\tau-\tau_{c}}{N \tau}\right] \theta^{2}+\frac{B}{N} \theta^{3}+\frac{C \tau_{c}}{N \tau} \xi_{0}^{2}(\nabla \theta)^{2}+\cdots
$$

where $F$ is an energy per unit cell, $A, B, C$ are numerical constants and $\boldsymbol{\theta}$ is the average of $\mathbf{S}_{\boldsymbol{i}}$.

Eq. (3.6) is justified as follows :

a) the terms

$$
\frac{A}{N} \theta^{2}+\frac{B}{N} \theta^{3}+\cdots
$$

come from the entropy of partially alined spins. The $1 / N$ factor expresses the fact that all spins in one chain are in unison. The existence of an $\theta^{3}$ term is related to the lack of centrosymmetry of the basic polyhedron (for $s \neq 2$ );

$b)$ the term $-\frac{A \tau_{\mathrm{c}} \theta^{2}}{N}$ describes the $J$ interaction between neighbour chains $\left(\tau_{\mathrm{c}} / N\right.$ is proportional to $\left.J\right)$;

$c$ ) the coefficient of the gradient term $(\nabla \theta)^{2}$ can be understood as follows : two neighbouring sites (i) and $(j)$ belonging to different chains have a difference of magnetization

$$
\theta_{i}-\theta_{j} \sim \nabla \theta \cdot\left(\overline{\mathbf{R}}_{(i)}-\overline{\mathbf{R}}_{(j)}\right)
$$

where $\bar{R}_{i}$ is the center of gravity of the chain to which (i) belongs, and :

$$
\left\langle\left(\bar{R}_{(i)}-\bar{R}_{(j)}\right)^{2}\right\rangle \sim \xi_{0}^{2} .
$$

We may eliminate $\tau$ and write the free energy in terms of $\theta$ and of the percolation probability $p$ (eq. (3.5)) :

$$
\frac{F}{\tau p_{\mathrm{c}}}=A \theta^{2} \frac{p_{\mathrm{c}}-p}{p_{\mathrm{c}}}+B \theta^{3}+C \xi_{0}^{2}(\nabla \theta)^{2} .
$$

Eq. (3.8) leads to a critical behaviour in agreement with the tree approximations : for instance when $p>p_{\mathrm{c}}$ the optimum of $F$ corresponds to

$$
\theta=\frac{2 A}{3 B} \frac{p-p_{\mathrm{c}}}{p_{\mathrm{c}}} \cong\left(\rho-\rho_{\mathrm{c}}\right) / \rho_{\mathrm{c}}
$$

Starting from eq. (3.8) it is easy to calculate the fluctuations of $\theta$ in the mean field approximation, and to apply the Ginsburg approach [5] : the results coincide with eq. $(2 \cdot 6-8)$.

Remark. - In the transition from the hamiltonian $\mathcal{H}$ to the mean field free energy $F$, we ignored any complication related to the disorder of the chains, or the resulting disorder of the coupling constants $J_{i j}$. In certain systems, a weak randomness in the coupling constants is enough to alter the critical exponents [15]. This complication is not expected to occur here, since the specific heat is not singular for the $s=0$ Potts model.

4. Conclusions. - For very long chains, we expect that the sol-gel transition induced by a weak crosslinking, to follow the classical picture, with the critical exponents (1.4). For shorter chains, we predict a cross over between classical behaviour, not too close from threshold and percolation behaviour (eq. 1.1-3), very close to threshold.

The fact that cyclisations become less important for long chains is intuitively obvious. But the merit of the Ginsburg approach is to tell us exactly how long the chains must be if we want the classical theory to apply at a given (small) distance from threshold : this is given by eq. (2.9).

Precise measurements of the weight average molecular mass $(Z)$ below threshold and of the gel fraction $(\theta)$ above threshold, for various chain lengths $(N)$ are not currently available, but would be of great interest.

\section{References}

[1] Flory, P., J. Amer. Chem. Soc. 63 (1941) 3083, 3091.

[2] Stockmayer, W., J. Chem. Phys. 11 (1943) 45.

Gordon, M. and SCantelbury, G., Trans. Faraday Soc. 60 (1964) 604.

[3] Flory, P., J. Am. Chem. Soc. 63 (1941) 3097.
[4] FloRY, P., Principles of polymer chemistry (Cornell Univ. Press, N.Y.), 1953, 1971.

[5] Stauffer, D., J. Chem. Soc., Faraday trans. 272 (1976) 1354.

[6] De Gennes, P. G., J. Physique Lett. 37 (1976) L-1. 
[7] Kirkpatrick, S., Rev. Mod. Phys. 45 (1973) 574.

Essam, J., in Phase transitions and critical phenomena, Eds C. Domb, M. Green (Academic Press N.Y.) 2 (1972) 197.

[8] De Gennes, P. G., J. Physique 36 (1975) 1049.

[9] For an introduction to the Ginsburg idea, see for instance appendix 2 of reference [5].

[10] CotTon, J. P. et al., Macromolecules 7 (1974) 863. KIRSTE, R. et al., Polymer 16 (1970) 120.
[11] Toulouse, G. and Pfeuty, P., Introduction aux groupes de renormalisation (Presses Univ., Grenoble) 1975.

[12] Potrs, R., Proc. Cambridge Philos. Soc. 48 (1952) 106.

[13] Fortuin, C. and Kastelejn, P., Physica 57 (1972) 536.

[14] Harris, A. B., Lubensky, T., Holcomb, W. and Dasgupta, C., Phys. Rev. Lett. 35 (1975) 327.

[15] Brooks-Harris, A. and Lubensky, T., Phys. Rev. Lett. 33 (1974) 1540. 